\title{
(1)
}

\section{Water Stress Responses of Tomato Mutants Impaired in Hormone Biosynthesis Reveal Abscisic Acid, Jasmonic Acid and Salicylic Acid Interactions}

\author{
Valeria A. Muñoz-Espinoza ${ }^{1 \dagger}$, María F. López-Climent ${ }^{1 \dagger}$, José A. Casaretto ${ }^{2}$ and \\ Aurelio Gómez-Cadenas ${ }^{1 *}$ \\ ${ }^{1}$ Departament de Ciències Agràries i del Medi Natural, Universitat Jaume I, Castelló de la Plana, Spain, ${ }^{2}$ Department of \\ Molecular and Cellular Biology, University of Guelph, Guelph, ON, Canada
}

\section{OPEN ACCESS}

Edited by:

Shabir Hussain Wani,

Sher-E-Kashmir University of Agricultural Sciences and Technology of Kashmir, India

Reviewed by: Vicente Martinez,

Centro de Edafología y Biología Aplicada del Segura - CSIC, Spain

Biswapriya Biswavas Misra,

University of Florida, USA

${ }^{*}$ Correspondence:

Aurelio Gómez-Cadenas aurelio.gomez@uji.es

${ }^{\dagger}$ These authors have contributed equally to this work

Specialty section: This article was submitted to

Plant Biotechnology,

a section of the journal

Frontiers in Plant Science

Received: 07 September 2015

Accepted: 30 October 2015

Published: 18 November 2015

Citation:

Muñoz-Espinoza VA,

López-Climent MF, Casaretto JA and Gómez-Cadenas A (2015) Water

Stress Responses of Tomato Mutants Impaired in Hormone Biosynthesis Reveal Abscisic Acid, Jasmonic Acid and Salicylic Acid Interactions.

Front. Plant Sci. 6:997. doi: 10.3389/fpls.2015.00997
To investigate the putative crosstalk between JA and ABA in Solanum lycopersicum plants in response to drought, suppressor of prosystemin-mediated responses2 (spr2, JA-deficient) and flacca (flc, ABA-deficient) mutants together with the naphthalene/salicylate hydroxylase (NahG) transgenic (SA-deficient) line were used. Hormone profiling and gene expression of key enzymes in ABA, JA and SA biosynthesis were analyzed during early stages of drought. ABA accumulation was comparable in spr2 and wild type (WT) plants whereas expression of 9-cis-epoxycarotenoid dioxygenase 1 (NCED1) and NCED2 was different, implying a compensation mechanism between NCED genes and an organ-specific regulation of NCED1 expression. JA levels and 12-oxo-phytodienoic acid reductase 3 (OPR3) expression in flc plants suggest that ABA regulates the induction of the OPR3 gene in roots. By contrast, $A B A$ treatment to flc plants leads to a reduction of JA and SA contents. Furthermore, different pattern of SA accumulation (and expression of isochorismate synthase and phenylalanine ammonia lyase 1) was observed between WT seedlings and mutants, suggesting that SA plays an important role on the early response of tomato plants to drought and also that JA and ABA modulate its biosynthesis. Finally, hormone profiling in spr2 and NahG plants indicate a crosstalk between JA and SA that could enhance tolerance of tomato to water stress.

Keywords: drought, flacca, hormonal crosstalk, Solanum Lycopersicum, spr2

\section{INTRODUCTION}

Drought is by far the most important environmental constrain in agriculture and every year the number of economic losses due to water constraints increases (Vicente-Serrano, 2007). This problem has been aggravated in the last decade by global climate changes.

Plants have developed a range of strategies to reduce the negative effects of drought on their physiology (Fang and Xiong, 2015). A complex network of highly coordinated hormonal interactions seems to be crucial for this process (Smekalova et al., 2014). Classically, the role of phytohormones have been described considering individual signaling pathways but this approach 
does not address the spatiotemporal specificity, considered central to fine-tune hormone signaling. Thus, the study of hormone profiling during early stages of water stress conditions in different mutant backgrounds could help to distinguish common from specific responses.

Perception of stress signals often causes changes in levels of different hormones to adapt and respond to environmental challenges (Shakirova et al., 2003; De Ollas et al., 2013, 2015; Rodríguez-Álvarez et al., 2015). Among phytohormones, abscisic acid (ABA), jasmonic acid (JA) and salicylic acid (SA) are known to play important roles in plant responses to stress.

Classically, ABA has been mainly related to abiotic stress (Gómez-Cadenas et al., 2002; Fujita et al., 2011). Structurally, $\mathrm{ABA}$ is a sesquiterpene derived from $\mathrm{C}_{40}$ oxygenated carotenoids with a number of steps controlling its synthesis. One of the key steps includes the cleavage of 9-cis-violaxanthin and/or 9-cis-neoxanthin to produce xanthoxin (North et al., 2007), a reaction catalyzed by 9-cis-epoxycarotenoid dioxygenase (NCED). Another important step is the oxidation of abscisic aldehyde by an abscisic aldehyde oxidase (AAO) which needs a molybdenum cofactor (MoCo) for its activity (Taylor et al., 1988).

By contrast, JA and SA have been considered signals of biotic stress threats for years because they fulfill essential roles in plant defense ( $\mathrm{Li}$ et al., 2003). JA derives from linolenic acid via the octadecanoid pathway. There are several enzymes involved in this pathway, including lipoxygenase (LOX), allene oxide synthase (AOS), allene oxide cyclase (AOC) and 12-oxophytodienoic acid reductase (OPR). Although the function of these enzymes in JA biosynthesis under biotic stress situations is well understood (reviewed in Wasternack and Hause, 2013), only recent information is found on their role under drought. Indeed, research on the molecular mechanisms underlying hormonal regulation in response to drought has uncovered a complex and dynamic regulatory network in which JA and ABA participate (Harb et al., 2010; Brossa et al., 2011; Savchenko et al., 2014).

It is well-known that SA is involved in a broad range of physiological and metabolic responses in plants (Hayat et al., 2010). This phenolic compound can be synthesized from the primary metabolite chorismate via two distinct enzymatic pathways, one involving phenylalanine ammonia lyase (PAL) and the other isochorismate synthase (ICS; reviewed in Chen et al., 2009). Szepesi et al. (2009) claimed that osmotic adaptation in tomato depends on SA concentration and also highlighted the difficulties in experimentally recreating the fine-balance and timing for the endogenous levels of the hormone. For instance, when applied at low concentrations, SA and JA acted synergistically, whereas a high concentration of one hormone antagonized the other one (Mur et al., 2006).

Hormonal deficient mutants are an interesting model system to study hormonal interactions. In tomato, ABA-deficient mutants such as sitiens (Taylor et al., 1988), flacca (flc, Sagi et al., 1999) and notabilis (Burbidge et al., 1999) and JA-deficient genotypes such as defenless 1 and suppressor of prosystemin-mediated responses 2 (spr2, Li et al., 2003) have been described. Spr2 plants are impaired in a fatty acid desaturase required for JA biosynthesis whereas flc plants have limited ABA biosynthesis due to impairment in a MoCo sulfurase, a cofactor needed for AAO function. Salicylic acid-deficient NahG (naphthalene/salicylate hydroxylase) transgenic line has been also used (Jia et al., 2013). It overexpresses the NahG gen from Pseudomonas putida that degrades SA to catechol, showing low levels of this hormone. Previous research with these mutants indicated an enhanced basal resistance to biotic factors mediated by SA (Audenaert et al., 2002; Avila et al., 2012), which suggest that SA can also interact with ABA and JA in the physiological responses to stress conditions (De Torres Zabala et al., 2009).

The research presented in this paper was designed to better understand the crosstalk between JA, SA, and ABA in tomato plants in response to drought. For this purpose, different genotypes with impaired production of hormones were used. Data indicate that not only JA and ABA are part of the signaling network controlling the responses of tomato plants to water stress conditions, but also SA is relevant.

\section{MATERIALS AND METHODS}

\section{Plant Material}

Seeds of Solanum lycopersicum L. were germinated in trays with vermiculite and after 1 month, they were transplanted to $2.5 \mathrm{~L}$ pots filled with perlite as a substrate. The following genotypes were used: spr2, $f l c, N a h G$ and the respective wild-type (WT) cultivars: Castlemar (CSL), Ailsa Craig (AC), and Moneymaker (MM). Plants were watered three times a week with $0.5 \mathrm{~L}$ of a half-strength Hoagland solution and allowed to acclimate for 3 months in a greenhouse with a $16 \mathrm{~h}$ photoperiod (maximum PAR $1200 \mu \mathrm{m} \mathrm{m}^{-2} \mathrm{~s}^{-1}$ ), $26 \pm 4{ }^{\circ} \mathrm{C}$ day temperature, $18 \pm 3{ }^{\circ} \mathrm{C}$ night temperature, and relative humidity ranging from 60 to $90 \%$. Four-month-old plants were used for all the experiments.

\section{Water Stress Conditions}

After the acclimation period, 20 plants of each genotype (AC, CSL, MM, spr2, flc, and NahG) were randomly distributed. Drought stress was imposed by transplanting plants to dry perlite (controls were transplanted to wet perlite at the same time). Within each group, leaves (from an intermediate position in the shoot) and young roots from four plants were collected at 0,1 , 3,6 , and $24 \mathrm{~h}$ after imposing the stress conditions. Tissue was immediately frozen in liquid nitrogen and stored at $-80^{\circ} \mathrm{C}$ until further analyses. For each of analyses described below (plant hormone profiling and RNA extraction), three independent extractions per group were performed.

\section{ABA Application}

A different group of $32 \mathrm{flc}$ plants was used for ABA treatment. Half of the plants, randomly distributed, was treated with a $10 \mu \mathrm{M}$ ABA solution (Sigma Aldrich, Madrid, Spain) whereas the other was watered with a mock solution as in (De Ollas et al., 2013). Preliminary experiments indicated that this ABA concentration had an effect on gene expression without having toxic effects on plants (data not shown). After 1 week of treatment, water stress was applied in both groups of plants as described above. Plants were collected at 0, 3, 6, and $24 \mathrm{~h}$ after initiating drought stress. 


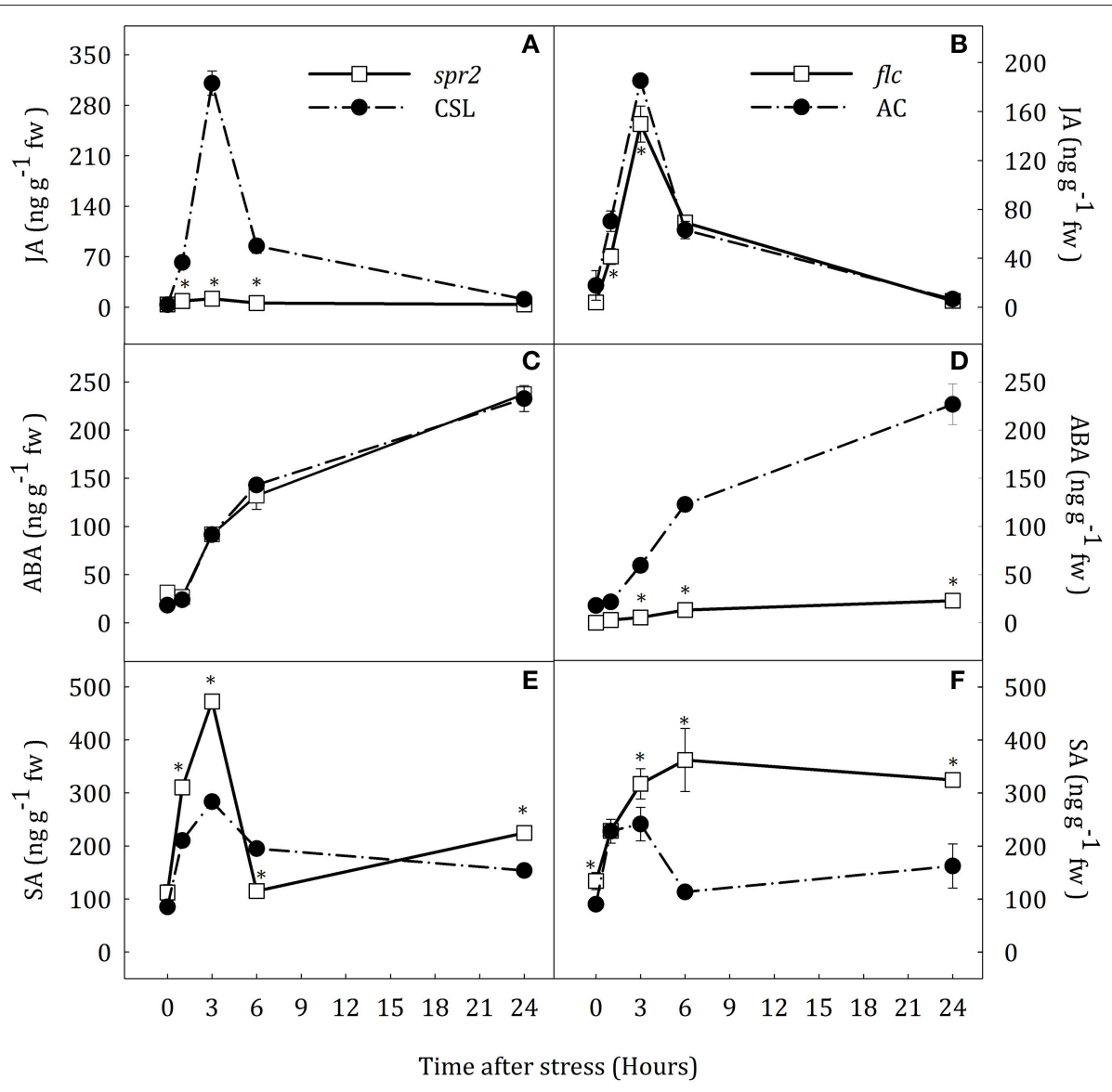

FIGURE 1 | JA (A,B), ABA (C,D) and SA (E,F) levels in roots of Solanum lycopersicum [WT (CSL and AC, black circles) and mutants (spr2 and flc, white square)] under control $(t=0)$ and water-stress conditions. Data are mean values \pm standard deviation of three independent determinations. Asterisks denote statistical difference with respect to WT at $p \leq 0.05$.

\section{Leaf Relative Water Content}

Plant water status was determined at each time point by measuring the relative water content (RWC) (Turner, 1981). After sampling, leaf fresh weights (FW) were determined, and then leaves were hydrated with distilled water for $24 \mathrm{~h}$ at ambient temperature in darkness until saturation. The leaves were then reweighed to obtained leaf turgid weights (TW). Subsequently, leaves were oven dried at $60^{\circ} \mathrm{C}$ for 3 days and their dry weights determined (DW). Leaf RWC was calculated following the formula; RWC $\%=(\mathrm{FW}-\mathrm{DW}) /(\mathrm{TW}-\mathrm{DW})^{*} 100$.

\section{Chlorophyll Fluorescence Measurements}

Quantum yield ( PPSII) measurements were performed with an OS 1 FL portable fluorometer (Opti-Sciences, Tyngsboro, MA, USA) in 12 light-adapted leaves per plant. Four plants per genotype were randomly chosen as replicates (López-Climent et al., 2008).

\section{Hormone Analysis}

Hormone extraction and analysis were carried out following the procedure described in Durgbanshi et al. (2005) with slight modifications. Frozen fresh tissue $(0,2 \mathrm{~g})$ was spiked with 100 ng of $\mathrm{d}_{6}-\mathrm{ABA}, 100 \mathrm{ng}$ of dihydrojasmonic acid and $100 \mathrm{ng}$ of $\mathrm{d}_{6}$-SA (prepared as in Gómez-Cadenas et al. (2002) and homogenized with $5 \mathrm{ml}$ of distilled water. After centrifugation at $4000 \times \mathrm{g}$ at $4{ }^{\circ} \mathrm{C}$, supernatants were recovered and $\mathrm{pH}$ adjusted to 3 with $30 \%$ acetic acid. The acidified water extract was partitioned twice against $3 \mathrm{ml}$ of diethyl ether. The organic upper layer was recovered and vacuum evaporated in a centrifuge concentrator (Speed Vac, Jouan, Saint HerblainCedex, France). The dry residue was then resuspended in a $10 \% \mathrm{MeOH}$ solution by gentle sonication. The resulting solution was passed through $0.22 \mu \mathrm{m}$ regenerated cellulose membrane syringe filters (Albet S.A., Barcelona, Spain) and directly injected into a UPLC system (Acquity SDS, Waters Corp., Milford, MA, USA).

Analytes were separated by reversed-phase (Nucleodur C18, $1.8 \mu \mathrm{m} 50 \times 2.0 \mathrm{~mm}$, Macherey- Nagel, Barcelona, España) using a linear gradient of ultrapure $\mathrm{H}_{2} \mathrm{O}(\mathrm{A})$ and $\mathrm{MeOH}(\mathrm{B})$ (both supplemented with $0.01 \%$ acetic acid) at a flow rate of $300 \mu \mathrm{l} \mathrm{min}^{-1}$. The gradient used was: (0-2 min) 90:10 (A:B), (2-6 min) 10:90 (A:B), and (2-6-7 min) 90:10 (A:B). Hormones were quantified with a Quattro LC triple quadrupole mass spectrometer (Micromass, Manchester, UK) connected online to the output of the column through an orthogonal Z-spray 


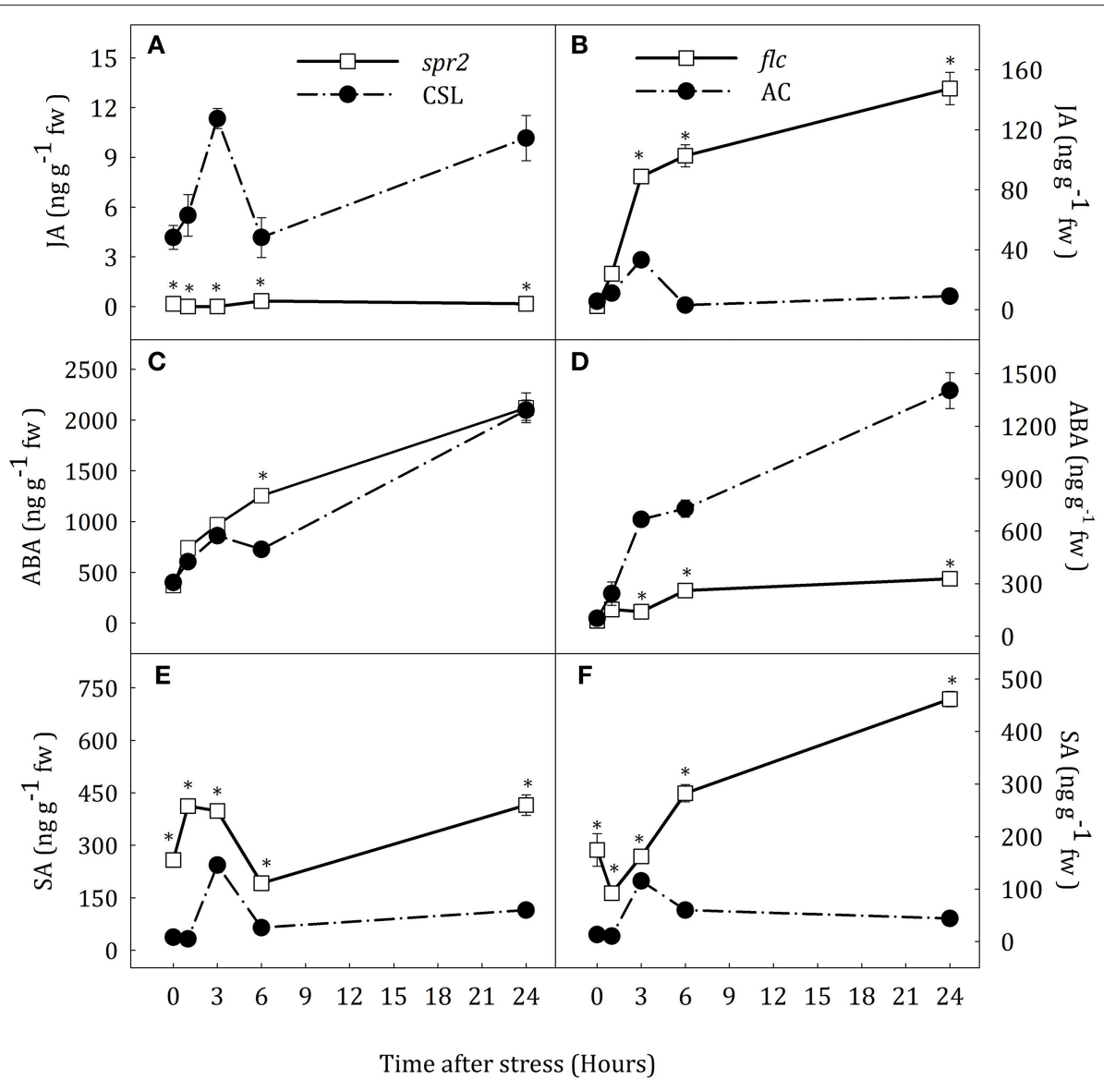

FIGURE 2 | JA (A,B), ABA (C,D) and SA (E,F) endogenous concentrations in leaves of Solanum lycopersicum [WT (CSL and AC, black circles) and mutants (spr2 and $f l c$, white square)] under control $(t=0)$ and water-stress conditions. Data are mean values \pm standard deviation of three independent determinations. Asterisks denote statistical difference with respect to WT at $p \leq 0.05$.

electrospray ion source. Quantitation of plant hormones was achieved by external calibration with standards of known amount.

\section{RNA Extraction and cDNA Synthesis}

Total RNA was extracted by using the E.Z.N.A. Kit (OMEGA Bio-Tek, Norcross, GA, USA) from frozen plant material according to the manufacturer's instructions. RNA integrity was visualized in $1 \%$ agarose gels while concentration and purity (260/280 ratio) was determined with a NanoDrop 2000 spectrophotometer (Thermo Scientific, Waltham, MA, USA). RNA was treated with DNase I (Fermentas, Hanover, MD, USA) to remove DNA contamination. First-strand cDNA was carried out using $2 \mu \mathrm{g}$ total RNA with the PrimerScript RT-PCR kit from Takara (Condalab, Barcelona, Spain) following the manufacture's protocol.

\section{Real-time qRT-PCR analysis}

Real-time qRT-PCR was performed using an ABI StepOne Detection System (Applied Biosystems, USA) and the Maxima SYBR Green/ROX qPCR mix from Fermentas (Thermo Scientific Fermentas, Spain). The primer details for the qRT-PCR are provided as supporting data (Table S1). Thermocycling conditions were: $95^{\circ} \mathrm{C}$ for $10 \mathrm{~min}$ followed by 40 cycles of $95^{\circ} \mathrm{C}$ for $10 \mathrm{~s}, 60^{\circ} \mathrm{C}$ for $10 \mathrm{~s}$ and $72^{\circ} \mathrm{C}$ for $20 \mathrm{~s}$. Fluorescent intensity data were acquired during the extension time. The specificity of the reaction was verified by melting curve analysis and relative quantification of the expression was done using the Relative Expression Software Tool (REST, Pfaffl et al., 2002). Normalization was performed using the expression levels of the $G A D P H$ gene. Results are expressed as the average of three independent replicate $\pm \mathrm{SE}$.

\section{Statistical Analysis}

Statistical analyses were performed using SPSS-10 statistical software (SPSS Inc., Chicago, IL, USA). Differences between mutants and WT plants were compared by using the least significant difference (LSD) test $(P \leq 0.05)$.

\section{RESULTS}

\section{RWC and $\Phi$ PSII in spr2 and flc Plants}

To investigate whether the spr 2 and $f l c$ mutations had an effect on signaling events in the early stages of water stress, both mutants 


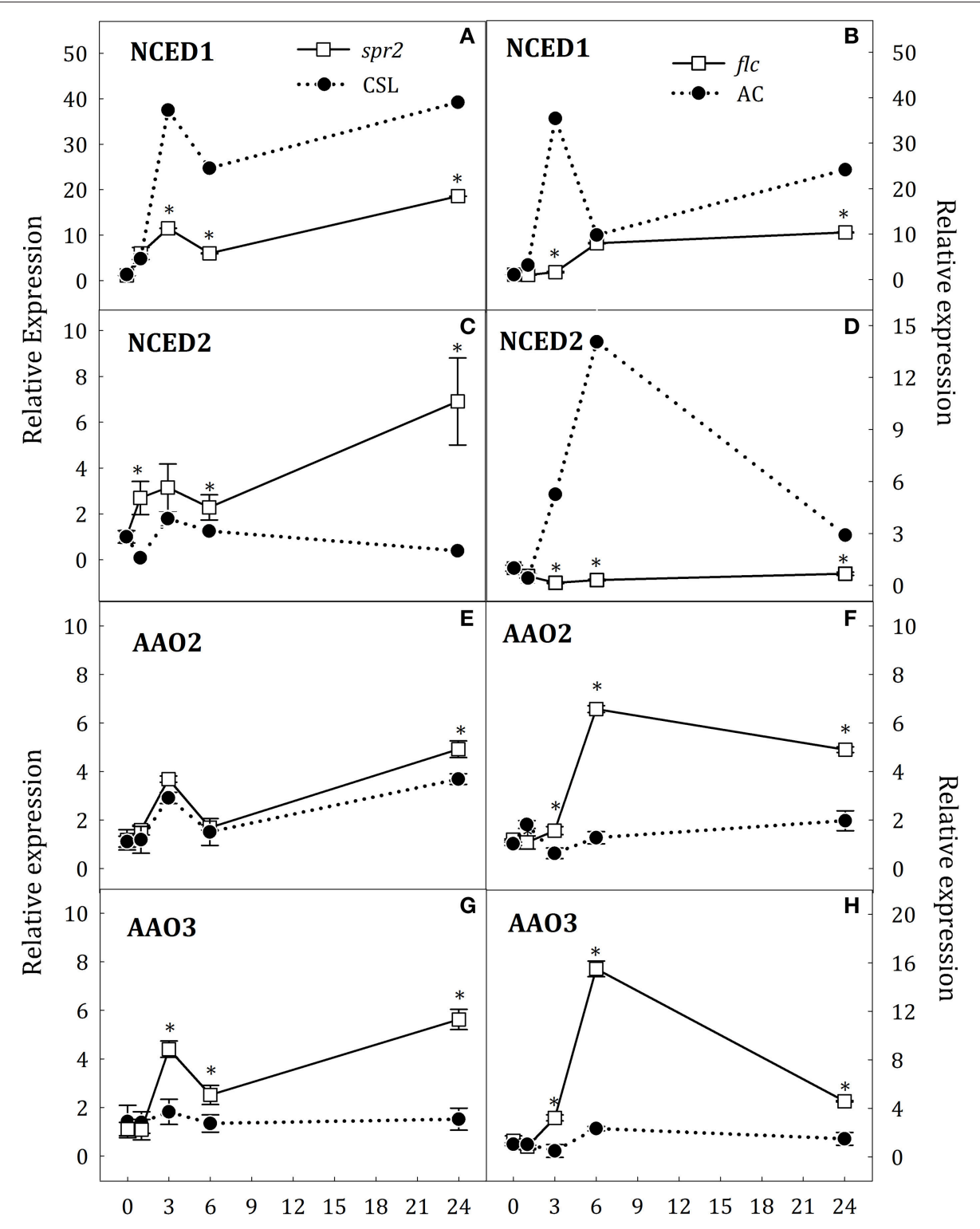

Time after stress (Hours)

FIGURE 3 | Relative expression of NCED1 (A,B), NCED2 (C,D), AAO2 (E,F), and AAO3 (G,H) in roots of Solanum lycopersicum [WT (CSL and AC, black circles) and mutants (spr2 and flc, white square)] under control $(t=0)$ and water-stress conditions. Data are mean values \pm standard deviation of three independent determinations. Asterisks denote statistical difference with respect to WT at $p \leq 0.05$.

were evaluated in comparison with WT, following a time-course experiment. Visible symptoms of leaf wilting were observed after stress imposition in WT and mutant plants. However, all plants recovered their turgid aspect when they were rehydrated after a 24 h-period of water stress (see Figure S1).

Differences in water content were evident after one $h$ of stress in all groups of plants. However, the $f l c$ mutant was the most affected (Figure S2). RWC decreased throughout the experimental period in the four groups of plants. Stress conditions caused an important loss of leaf water in spr2 and CSL plants, and RWC followed a similar pattern in both genotypes
(Figure S2A). At the end of experiment, the RWC reached the minimum value, 67.4 and $63.6 \%$, in spr2 and CSL, respectively. Compared to WT, flc plants lost $20 \%$ more water content after $6 \mathrm{~h}$ of stress (Figure S2B). This difference was similar after $24 \mathrm{~h}$ of water deprivation. The highest values of RWC were observed in $\mathrm{AC}$, suggesting a better capacity to regulate water status during the stress condition.

Water stress reduced $\Phi$ PSII in both groups of plants (Figures S2C,D). In CSL and spr2 plants, ФPSII only decreased at the end of experiment, with a reduction of $17.6 \%$ in CSL and $7.1 \%$ in spr 2 with respect control plants. However, no differences were 


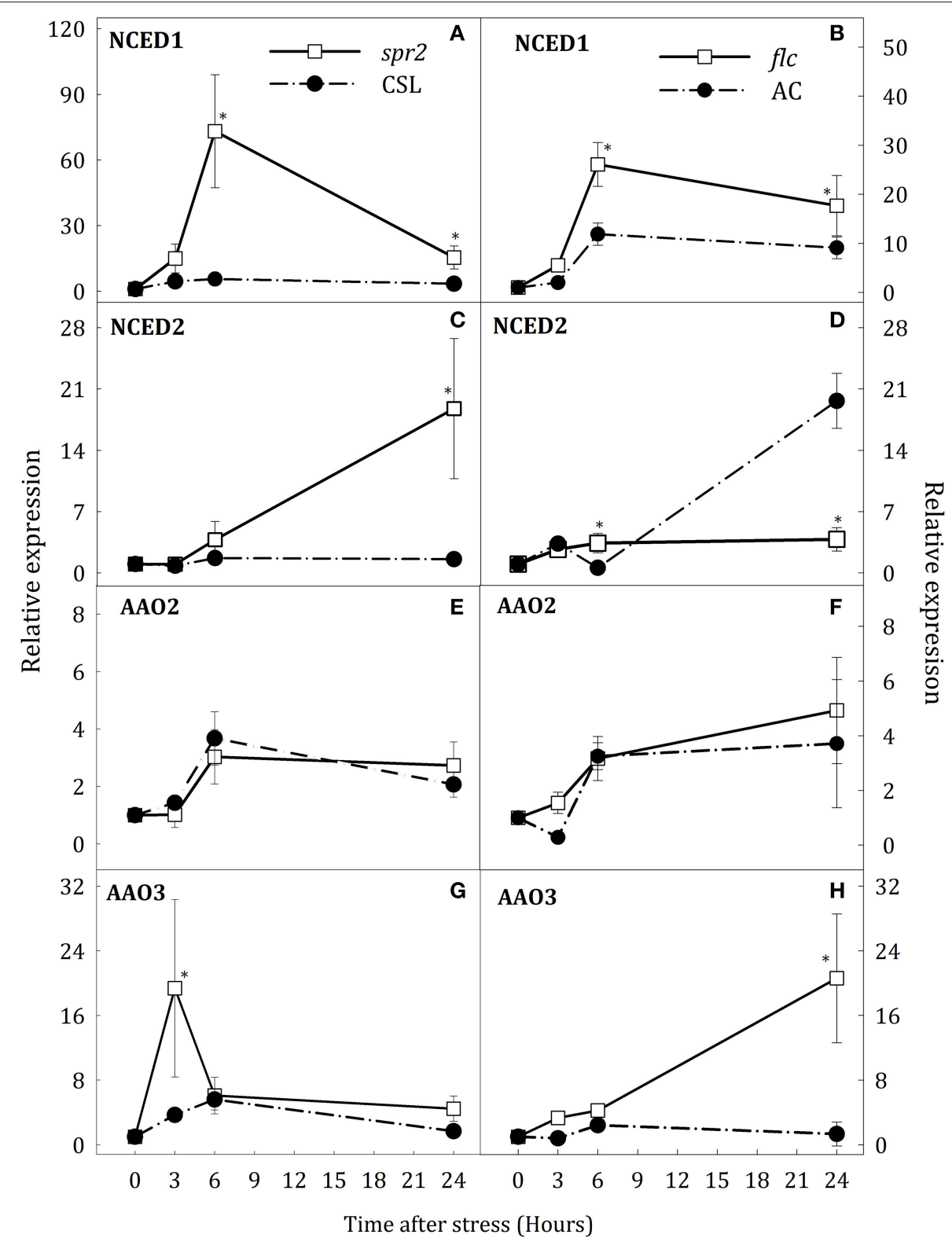

FIGURE 4 | Relative expression of NCED1 (A,B), NCED2 (C,D), AAO2 (E,F), and AAO3 (G,H) in leaves of Solanum lycopersicum [WT (CSL and AC, black circles) and mutants (spr2 and flc, white square)] under control $(t=0)$ and water-stress conditions. Data are mean values \pm standard deviation of three independent determinations. Asterisks denote statistical difference with respect to WT at $p \leq 0.05$.

observed between CSL and spr2. In contrast, ФPSII considerably decreased after $3 \mathrm{~h}$ of water deprivation in $f l c$ mutants showing remarkable differences with respect to WT plants although $\Phi$ PSII values were similar in mutant and WT plants at the end of experiment.

\section{Hormonal Balance during Early Stages of Water Stress}

JA, ABA, and SA levels were determined as indicators of the hormonal status in roots and leaves of spr2 and flc mutants (Figures 1, 2).

\section{Roots}

Hormonal profiles were slightly different in roots of spr2 and flc mutants but similar between their respective WT plants (Figure 1). Water stress induced a rapid increase of endogenous JA concentration which reached maximum values at $3 \mathrm{~h}$ after the stress imposition in CSL, AC and flc plants (Figures 1A,B). JA content in $f l c$ was significantly lower than in $\mathrm{AC}$ at 3 and $6 \mathrm{~h}$. Interestingly, the same result was observed in similar experiments with notabilis mutants that have limited ABA biosynthesis due to a null mutation in the NCED1 gene (see Supplementary Data Figure S3A). After this transient increase, root JA content in stressed plants decreased reaching basal 


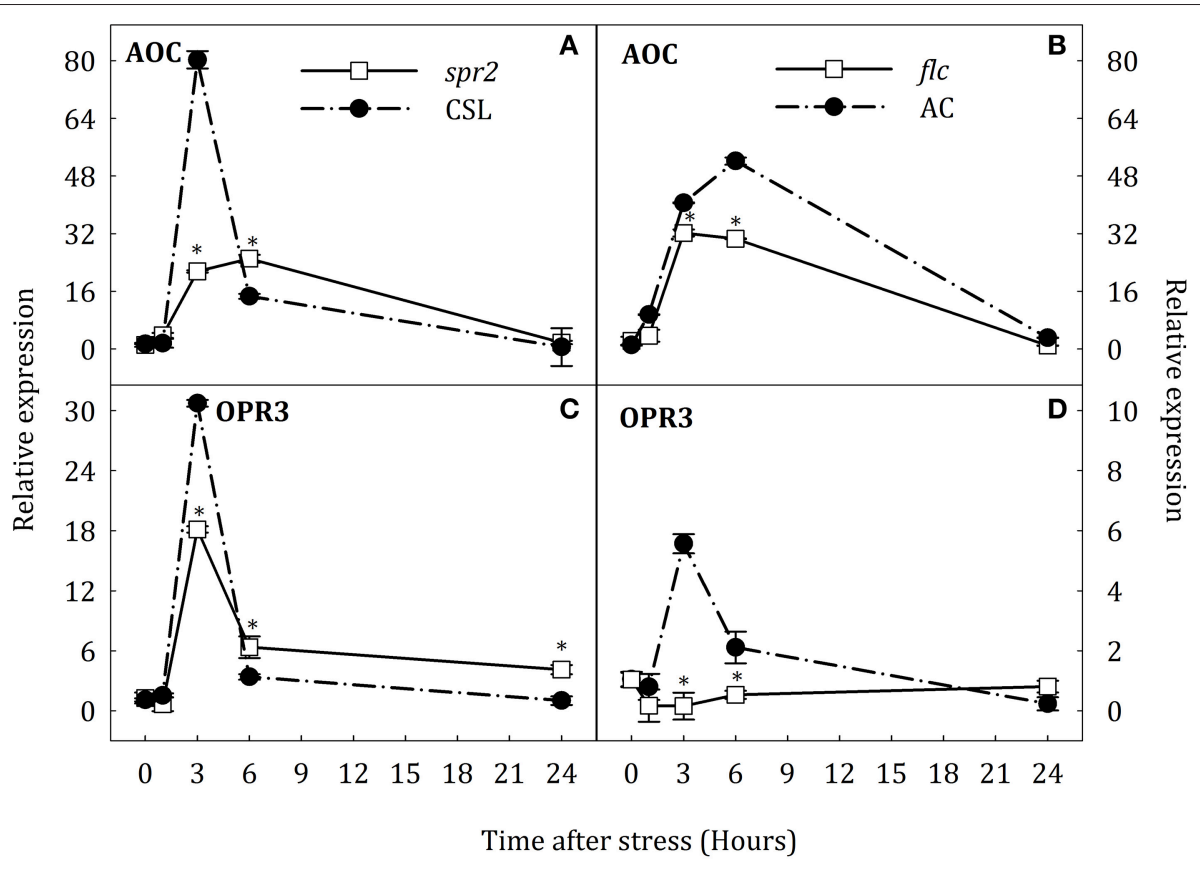

FIGURE 5 | Relative expression of AOC (A,B) and OPR3 (C,D) in roots of Solanum lycopersicum [WT (CSL and AC, black circles) and mutants (spr2 and flc, white square)] under control $(t=0)$ and water-stress conditions. Data are mean values \pm standard deviation of three independent determinations. Asterisks denote statistical difference with respect to WT at $p \leq 0.05$.

levels. Spr2 plants showed basal levels of JA throughout the experimental period (Figure 1A). The increase of ABA levels was progressive from $3 \mathrm{~h}$ in WT and spr2 plants, reaching values 12.6-fold higher than in well-watered plants at the end of the experiment (Figures 1C,D). Compared to other species, the lack of transient JA accumulation did not affect the progressive accumulation of ABA. As expected, flc plants showed basal levels of ABA throughout the experimental period (Figure 1D). Water stress induced a transient increase in SA concentration in CSL and AC plants (3.3- and 2.7-fold higher than levels in wellwatered plants at $3 \mathrm{~h}$ of stress; Figures 1E,F). In addition, spr2 and $f l c$ mutants showed different levels of SA accumulation. The increase of SA content in spr2 plants was 1.7-fold higher than in CSL plants. However, after $6 \mathrm{~h}$ of water stress, SA levels decreased to basal levels (Figure 1E). At the end of the experiment, SA levels in spr2 roots were slightly higher than in CSL (1.4-fold). On the contrary, SA concentration in $f l c$ mutants progressively increased from the beginning of the experiment, reaching levels 2.4-fold higher than in well-watered plants at the end of experimental period (Figure 1F).

\section{Leaves}

Hormone profiles in leaves of CSL and AC plants were similar to those in roots. Water deprivation caused a transient increase in JA and SA concentration at $3 \mathrm{~h}$ of stress and a progressive increase of ABA content (Figure 2). In leaves of spr2 plants, JA concentration was almost constant at basal levels. Again, ABA accumulation pattern in this JA-deficient mutant was similar to that in CSL (Figures 2A,C). In contrast, leaf JA content progressively increased in $f l c$ mutants, being 16.3 -fold higher than in $\mathrm{AC}$, at the end of the experiment. The endogenous concentration of ABA in $f l c$ plants was $76.6 \%$ lower than in $\mathrm{AC}$ seedlings at the end of experiment (Figures 2C,D). Similarly to that observed in roots, flc and spr2 mutants showed a different pattern of SA accumulation. Leaf SA levels of both mutants were higher than in their respective WT backgrounds throughout the experiment (Figures 2E,F). Spr2 plants showed a slight peak in SA content after the stress imposition, reaching maximum levels $3 \mathrm{~h}$ after the stress onset (1.6-fold increase with respect to the control levels). In $f l c$ mutants by contrast, SA levels progressively increased throughout the experimental period after a slight reduction. The highest concentration of SA was observed in spr2 and flc plants at the end of the experiment (Figures 2E,F).

Our data indicate that JA deficiency do not affect ABA accumulation during water stress. Furthermore, the alteration of SA and JA concentrations in response to water stress suggests an involvement of these hormones in signaling this condition.

\section{Gene Expression Pattern for Key Genes of JA and ABA Biosynthesis Pathways}

To determine whether altered levels of JA and ABA during water stress could also affect the expression of key genes for the biosynthesis of both hormones, gene expression was evaluated in spr2 and $f l c$ plants. Water deprivation strongly induced expression of NCED1 and slightly that of NCED2 in WT roots. NCED1 transcript levels were clearly down-regulated in roots of both mutants with respect to WT (Figures 3A,B). In spr2 plants, the increase of NCED2 transcript levels was higher than in CSL plants (17.8-fold higher at $24 \mathrm{~h}$, Figure 3C). By contrast, in flc plants, NCED2 expression was not induced throughout the 


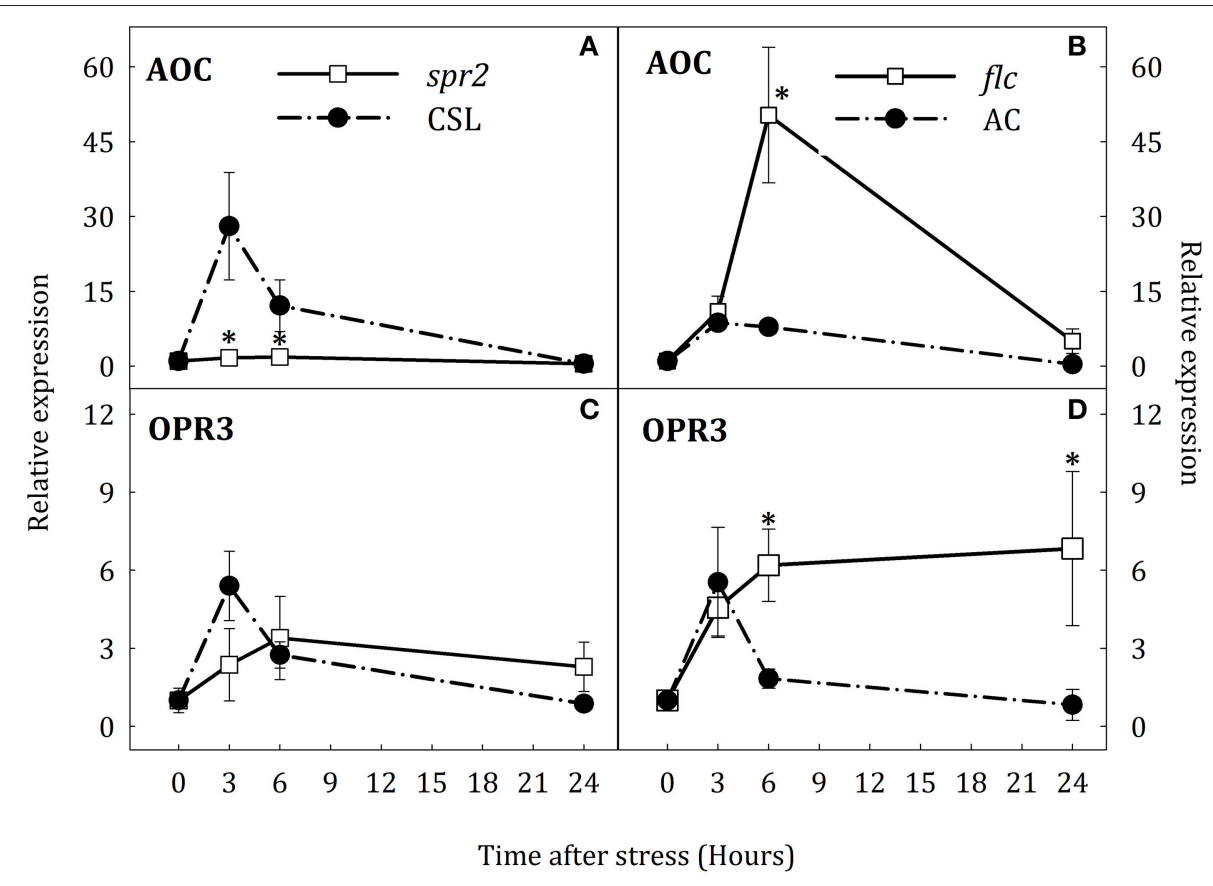

FIGURE 6 | Relative expression of AOC (A,B) and OPR3 (C,D) in leaves of Solanum lycopersicum [WT (CSL and AC, black circles) and mutants (spr2 and $f l c$, white square)] under control $(t=0)$ and water-stress conditions. Data are mean values \pm standard deviation of three independent determinations. Asterisks denote statistical difference with respect to WT at $p \leq 0.05$.

experimental period (Figure 3D). In leaves of stressed plants, NCED1 was up-regulated in spr2 and $f l c$ mutants after $6 \mathrm{~h}$ of stress (Figures 4A,B) whereas NCED2 transcripts were only different in both mutants with respect to WT at $24 \mathrm{~h}$ of stress imposition (Figures 4C,D). Again, as in roots, the trend was different in both mutants: NCED2 expression in spr2 was higher than in CSL and in $\mathrm{flc}$ lower than in AC.

Transcript levels of the three $A A O$ genes were also determined. In stressed roots, no changes in the expression of $A A O 1$ were observed and, therefore, data have been omitted. However, $A A O 2$ and $A A O 3$ were up-regulated in spr2 and $f l c$ mutants (Figures $3 \mathrm{E}-\mathbf{H}$ ). In spr2 plants, the increase of $A A O 2$ transcript levels was slightly higher than in WT but $A A O 3$ expression was significantly elevated (4.2-fold higher than in CSL plants at $24 \mathrm{~h}$, Figures 3E,G). Similar trends were observed in $f l c$ mutants (Figures $\mathbf{3 F}, \mathbf{H}$ ), as $A A O 2$ and $A A O 3$ transcript levels were strongly induced after $6 \mathrm{~h}$ of stress $(34.4$ and $73.2 \%$ higher than WT, respectively), and remained high at the end of experiment. In leaves, water stress conditions induced the expression $A A O 2$ and $A A O 3$ but, contrary to roots, scarce differences were observed between WT plants and mutants (Figures 4E-H). Levels of AAO2 transcripts were similar in mutants and WT as $A A O 2$ transcript abundance increased after $6 \mathrm{~h}$ of water stress (Figures 4E,F). However, AAO3 expression was slightly higher in stressed spr2 and flc plants than in WT (Figures 4G,H).

In response to stress, expression of $A O C$ and $O P R 3$ was induced in roots and leaves of WT plants (Figures 5, 6). In roots, $A O C$ expression in spr 2 and $f l c$ mutants was lower than in their respective WT genotypes after $3 \mathrm{~h}$ of stress (Figures 5A,B). OPR3 expression was induced at the same time in CSL, AC, and spr2 seedlings. In this case, OPR3 expression was lower in spr2 than in CSL (Figure 5C). Surprisingly, transcript levels of OPR3 did not increase in $f l c$ mutants under the stress condition, remaining above basal levels throughout the experiment (Figure 5D). In contrast, no major changes in the expression of $A O C$ were observed in leaves of $s p r 2$ plants although the expression of this gene, as in roots, was strongly induced in CSL after $3 \mathrm{~h}$ of stress (Figure 6A). In addition, the expression profile of OPR3 was similar in spr2 and CSL plants, showing a slight increase after $3 \mathrm{~h}$ of stress and returning to basal levels at $24 \mathrm{~h}$ (Figure 6C). In $\mathrm{flc}$ and AC leaves, $A O C$, and $O P R 3$ expression did not decrease after $6 \mathrm{~h}$ of stress (Figures 6B,D).

These results suggest an organ-specific regulation of NCED1 expression and compensative mechanisms to induce $\mathrm{ABA}$ accumulation in spr2 mutant. Furthermore, data indicate that SA may participate in ABA biosynthesis by induction of AAO3 expression. On the other hand, results obtained in $f l c$ mutants evidence that endogenous ABA levels may modulate OPR3 expression in roots and leaves.

\section{Gene Expression Pattern for Key Genes in SA Biosynthesis}

To test the hypothesis that SA accumulation under water stress could result from the novo biosynthesis of this hormone and that JA- and ABA- deficiency could affect the expression of key genes in the SA biosynthesis, the expression of ICS and PAL1 was evaluated by qRT-PCR in roots and leaves of all groups 


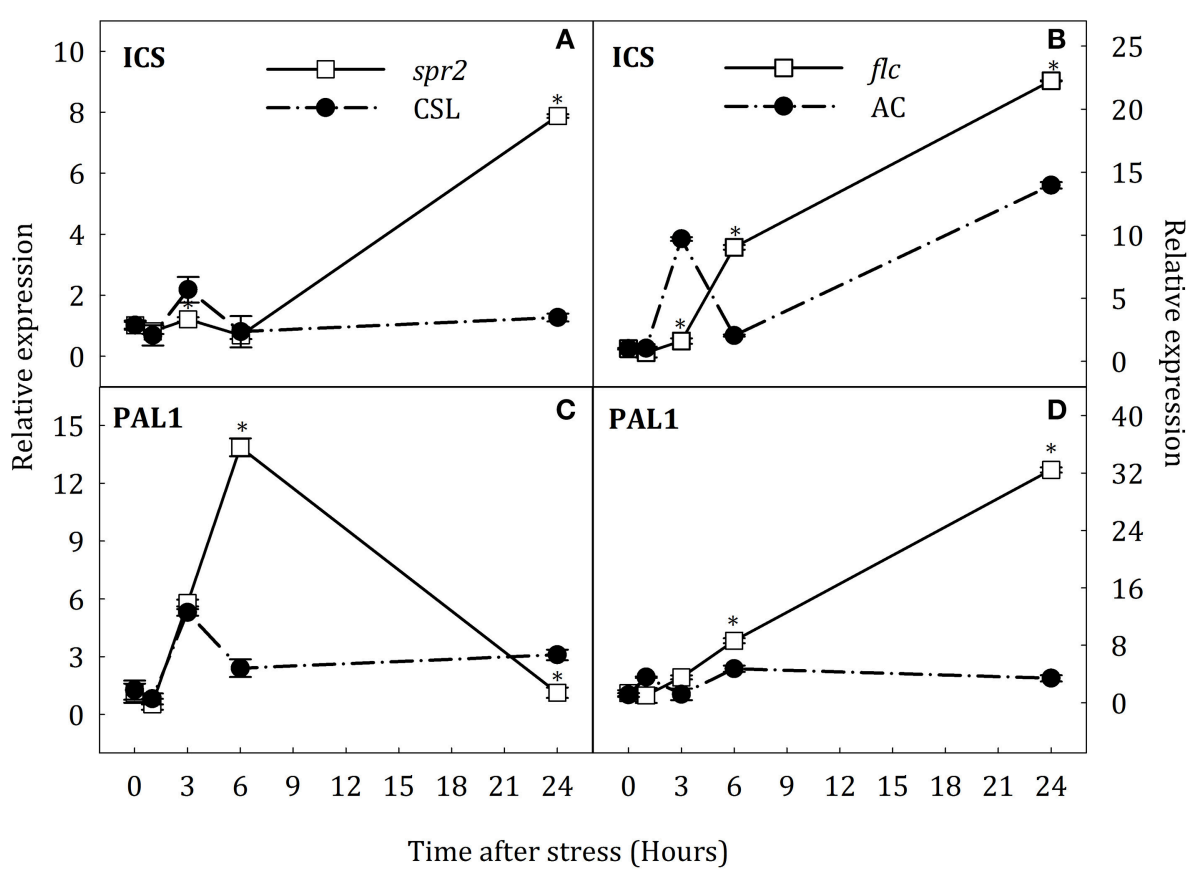

FIGURE 7 | Relative expression of ICS (A,B) and PAL1 (C,D) in roots of Solanum lycopersicum [WT (CSL and AC, black circles) and mutants (spr2 and flc, white square)] under control $(t=0)$ and water-stress conditions. Data are mean values \pm standard deviation of three independent determinations. Asterisks denote statistical difference with respect to WT at $p \leq 0.05$.

of plants. The expression of both genes in roots showed a transient up-regulation at the early stages of stress in $\mathrm{AC}$ and CSL plants (Figure 7). In spr2 mutants, ICS transcript levels considerably increased at the end of stress (8.2-fold higher than in CSL, Figure 7A). Moreover, PAL1 expression in spr2 mutants was twice as high as in CSL plants at $6 \mathrm{~h}$ of stress, reverting to control levels by the end of experiment (Figure 7C). In $f l c$ mutants, ICS and PAL1 transcripts increased after $6 \mathrm{~h}$ of stress, remaining higher than in $\mathrm{AC}$ at $24 \mathrm{~h}$ (1.6-fold for ICS and 9.4-fold for PAL1, with respect to WT, Figures 7B,D). In stressed leaves, ICS and PAL1 were down-regulated in spr2 plants (Figures 8A,C). In contrast, ICS transcripts increased at the end of the experiment in flc mutants (2.5-fold higher than in well-watered plants, Figure 8B) and PAL1 expression increased throughout the experimental period (Figure 8D). WT genotypes (CSL and AC) showed low levels of ICS and PAL1 transcripts throughout the experiment. These results indicate that accumulation of SA could result, at least in part, from de novo synthesis, by induction of ICS and PAL1 expression. They also point to an interaction of JA and ABA on SA biosynthesis.

\section{ABA Treatment in flc plants}

To test the effect of ABA on SA and JA levels, flc mutants were treated with $10 \mu \mathrm{M}$ ABA and thereafter exposed to water stress. Figure 9 shows that JA content remained on basal levels after ABA treatment in well-watered plants. On the contrary, SA levels decreased by the ABA treatment in roots and leaves (76.0 and 92.3\% of control values, respectively). Under water deprivation, the pattern of JA accumulation was similar in roots of both groups of plants, although ABA-treated plants showed lower levels at 3 and $24 \mathrm{~h}$ of stress (56.5 and $83.4 \%$ with respect levels in non-treated plants, respectively). In contrast, ABA treatment reduced leaf JA levels during water stress. Non-treated plants showed a drastic increased of JA content after the onset of stress whereas ABA-treated plants only showed a slight accumulation at 3 and $24 \mathrm{~h}$ of stress, with values 87.2 and $37.4 \%$ lower than in non-treated plants (Figure 9B). ABA treatment reduced SA concentration in roots and leaves throughout the experimental period, except in leaves after $3 \mathrm{~h}$ of stress (Figures 9C,D).

\section{Hormonal Profiles in a NahG Line under Water Stress Conditions}

Based on previous results obtained with spr2 and flc mutants, a transgenic NahG tomato line (cv. Moneymaker, MM) was used to evaluate the effect of SA deficiency on JA and ABA accumulation during water deprivation. Hormone profiles in MM plants under water deprivation were similar to those in AC and CSL plants (Figure 10). After $3 \mathrm{~h}$ of stress, MM plants showed a transient peak of JA and SA and also a progressive increase of ABA in both organs (Figure 10). In contrast, the pattern of JA and ABA accumulation in the NahG line was slightly different to that in WT. In roots of stressed NahG plants, JA content was higher than in MM (2.0- and 23.2-fold increase at 3 and $6 \mathrm{~h}$, respectively; Figure 10A). ABA levels were only different at the end of the experiment (2.8 fold increase with respect to MM, Figure 10C). As expected, SA remained at basal levels in $N a h G$ roots throughout the experiment (Figure 10E). The pattern of JA accumulation in leaves of stressed $N a h G$ plants was similar to that in roots (Figure 10B). The transient increase of JA levels in 


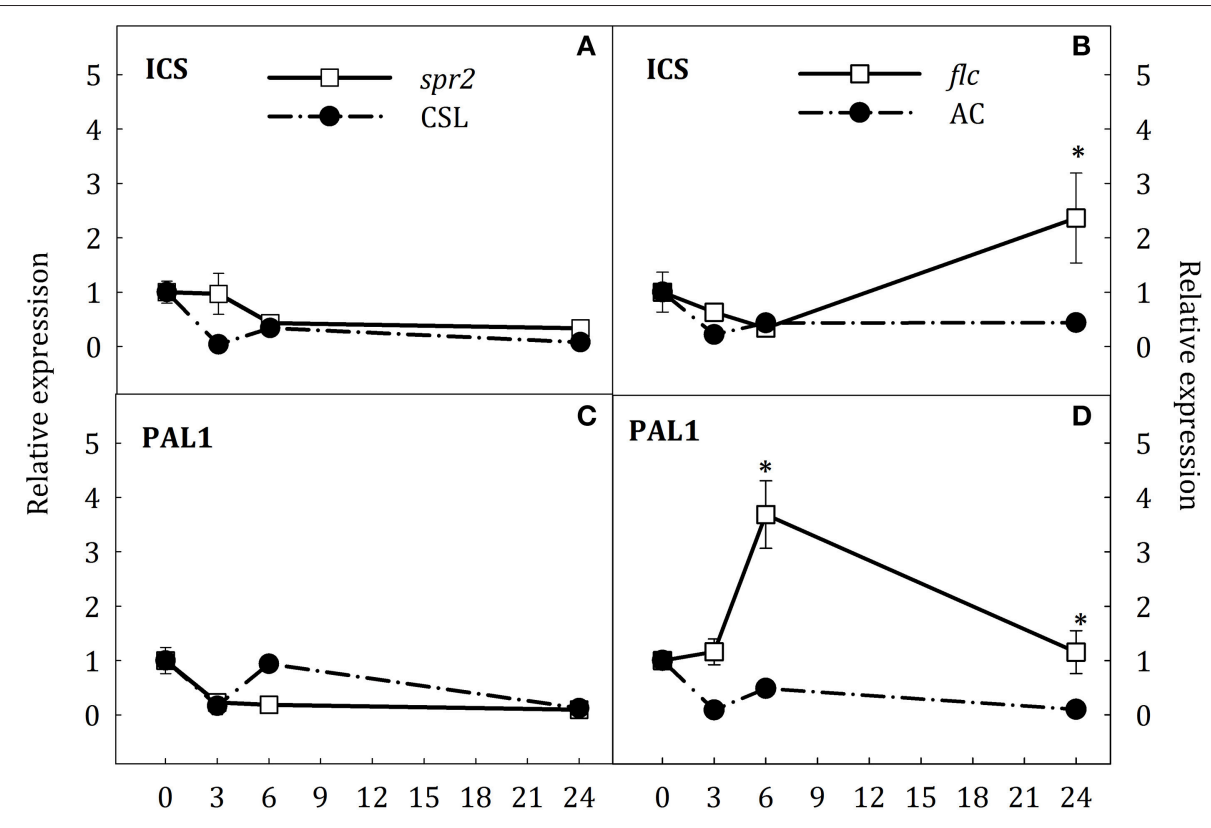

Time after stress (Hours)

FIGURE 8 | Relative expression of ICS (A,B) and PAL1 (C,D) in leaves of Solanum lycopersicum [WT (CSL and AC, black circles) and mutants (spr2 and flc, white square)] under control $(\boldsymbol{t}=\mathbf{0})$ and water-stress conditions. Data are mean values \pm standard deviation of three independent determinations. Asterisks denote statistical difference with respect to WT at $p \leq 0.05$.

these plants occurred after $6 \mathrm{~h}$ of stress whereas in MM plants this was observed at $3 \mathrm{~h}$ of stress. ABA accumulation in NahG leaves progressively increased after $6 \mathrm{~h}$ of stress, (not at $3 \mathrm{~h}$ as in $\mathrm{MM}$ ), and the ABA levels in the transgenic plants were $30.2 \%$ lower than in $\mathrm{MM}$ at the end of experiment. Under stress, SA concentration in NahG leaves was lower than in MM (Figure 10) although basal levels at time 0 were higher in the transgenic line. Therefore, the analysis of the NahG line clearly suggests a crosstalk between JA and SA under water stress situations.

\section{DISCUSSION}

Increasing number of recent reports in the literature point to the importance of JA on drought signaling (Brossa et al., 2011; Daszkowska-Golec and Szarejko, 2013; De Ollas et al., 2013, 2015); however crosstalk with other hormones remains unclear. A further layer of complexity arises from antagonistic and synergistic interactions between hormones and their tissuespecific biosynthesis in relation to signaling under water stress conditions (Seo and Koshiba, 2002; Kazan and Manners, 2008). The present work indicates that SA, along with JA and ABA, is involved in signaling water stress responses. Moreover, our data demonstrate that the expression of key genes for the biosynthesis of these three hormones is induced by drought and that regulation of JA and ABA levels differs in roots and leaves.

Despite the involvement of ABA and JA in drought signaling (Fujita et al., 2011; De Ollas et al., 2013, 2015), some reports also indicate that SA improves tolerance to water stress (Singh and Usha, 2003). To test the relation among JA, ABA, and SA, tomato mutants deficient in JA and ABA (spr2 and $f l c$ ) were used. Unlike previous research in Arabidopsis (Brossa et al., 2011), our data showed that JA-deficiency in tomato neither increased water loss nor modified $\Phi$ PSII with respect to WT seedlings. Moreover, contrary to what was observed by Brossa et al. (2011), the low levels of JA in stressed spr2 plants did not affect ABA accumulation in any of the organs analyzed.

To further analyze ABA biosynthesis during water stress, expression of key genes involved in this pathway was examined in spr2 and flc mutants. NCED and AAO genes typically form multigene families showing different organ-specific patterns of expression and environmental responsiveness. In tomato, only NCED1, NCED2, and NCED6 were known to be involved ABA biosynthesis (Taylor et al., 2005; Ntatsi et al., 2014). NCED1 is the orthologous member of the Arabidopsis (NCED3) which strongly respond to water stress (Iuchi et al., 2001) although other genes of this family also respond to osmotic and salinity stress. Therefore, when one gene is inactivated, another family member may take over its function. The low levels of NCED1 transcripts in spr 2 roots are consistent with previous studies showing that JA is required for its up-regulation (De Ollas et al., 2013, 2015; Du et al., 2014) under stress. However, despite the evident role of NCED1 as key enzyme in ABA biosynthesis under water stress, up-regulation of NCED2 in spr2 plants together with the high levels of endogenous ABA found in this mutant suggest a possible compensative mechanism for $\mathrm{ABA}$ biosynthesis in this genotype. In contrast, the transient increase of JA in flc mutant was quite similar to that in AC plants though levels of NCED1 transcripts were lower than in AC whereas NCED2 was not 


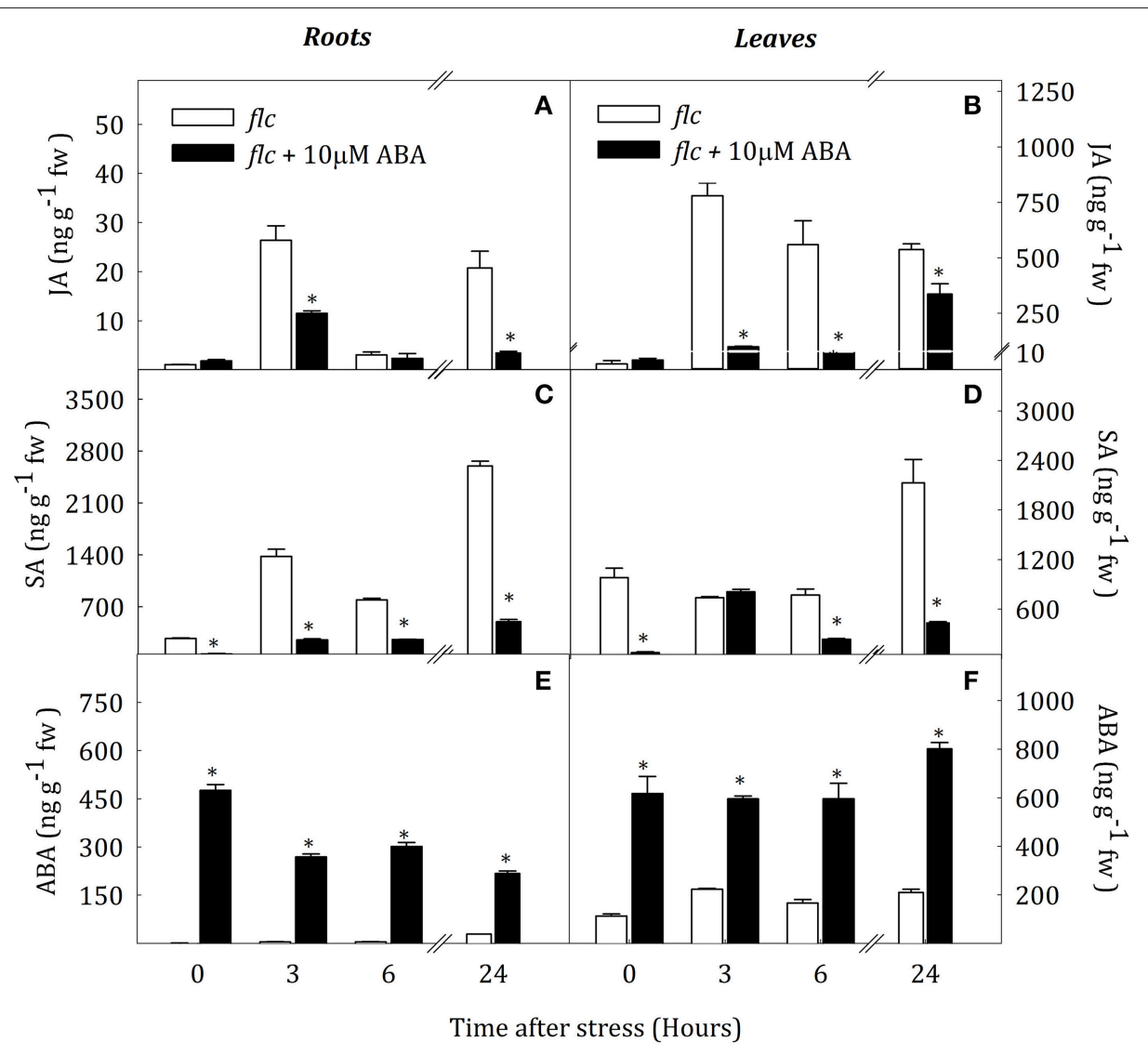

FIGURE 9 | JA (A,B), ABA (C,D) and SA (E,F) endogenous concentrations in leaves and roots of Solanum lycopersicum under control $(t=0)$ and water-stress conditions in flc seedlings without treatment (white bars) and flc seedlings and previously treated with $10 \mu \mathrm{M}$ ABA (black bars). Data are mean values \pm standard deviation of three independent determinations. Asterisks denote statistical difference with respect to WT at $p \leq 0.05$.

up-regulated. This could be explained, at least in part, because NCED1 expression might be negatively regulated by any of the intermediates in the ABA biosynthesis pathway. In this case, it is possible that $f l c$ mutant accumulated xanthoxin or abscisic aldehyde under water stress, considering the loss of function of AAO enzyme. Thus, we can speculate that these metabolites negatively affect the NCED1 expression.

Previous reports have shown that $A A O 3$ is the major $A A O$ gene involved in ABA biosynthesis in Arabidopsis (Seo et al., 2000) whereas in tomato $A A O 1, A A O 2$, and $A A O 3$ could perform this function. Data presented herein demonstrate that $A A O 2$ and $A A O 3$ are also induced by water stress in tomato roots. These results partially disagree with those of Yesbergenova et al. (2005) as they only describe an increase of AAO3 expression in roots; however, differences may be explained by the moderate stress applied in that study vs. the more severe drought condition imposed in our work. Additionally, it has been demonstrated that SA can increase $A A O$ expression (Szepesi et al., 2009). In this context, up-regulation of $A A O 2$ and $A A O 3$ in spr2 and $f l c$ mutants under water stress, concomitant with the increase of SA content, suggests that SA could be responsible for the positive regulation of $\mathrm{ABA}$ levels in tomato roots. The lack of a clear correlation between SA and ABA levels in NahG plants under water stress could be explained in terms of different strategies developed to compensate the SA deficiency.

Molecular and hormonal data obtained in roots and leaves of both mutants used indicate a complex, organ-specific regulation of JA and ABA pathways. The unexpected lack of induction of $O P R 3$ in $f l c$ and notabilis roots together with the high induction of OPR3 and the decreased of JA levels in flc leaves treated with $\mathrm{ABA}$, suggest that $\mathrm{ABA}$ is involved in OPR3 expression under water stress and their function differ from roots to leaves. In concordance with this result, a recent study in Arabidopsis (Savchenko et al., 2014) described a cooperative function of OPDA and ABA to control stomatal closure in response to drought, noticing the relevance of OPR3. Furthermore, previous reports have demonstrated the role of 12-OPDA in drought resistance (Seki et al., 2007; Grebner et al., 2013). Similar relation between JA and ABA was observed when NCED1 expression in both mutants was analyzed in roots and leaves. The lower induction of NCED1 in roots seems to be related to JA levels. However, both JA and ABA deficiency resulted in NCED1 overexpression in leaves, indicating that both hormones are able to reduce NCED1 transcript accumulation. In this line, a recent work in tomato (Du et al., 2014) indicated that 


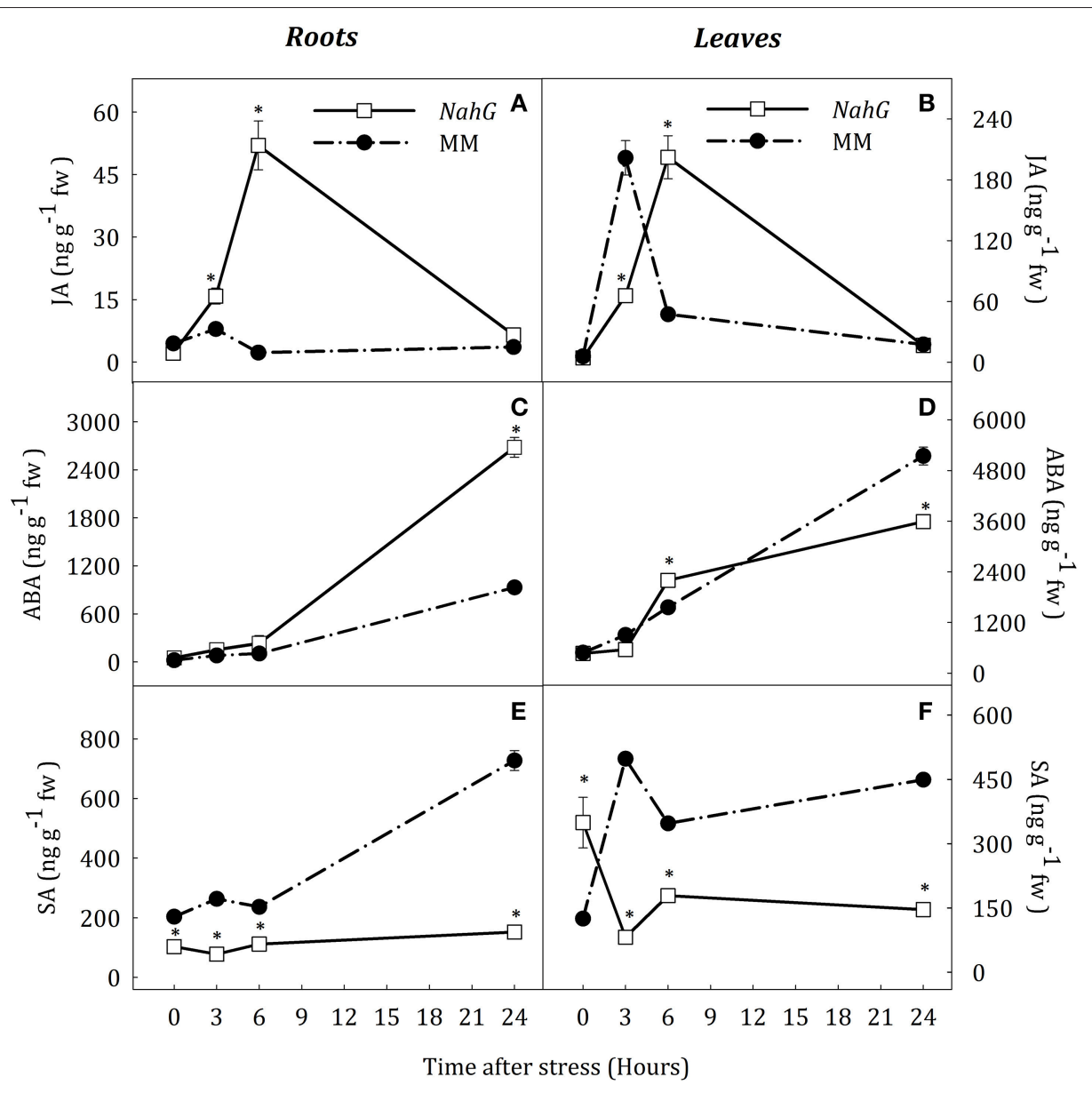

FIGURE 10 | JA (A,B), ABA (C,D) and SA (E,F) endogenous concentrations in leaves and roots of Solanum lycopersicum [WT (MM, black circles) and NahG line (white square)] under control $(t=0)$ and water-stress conditions. Data are mean values \pm standard deviation of three independent determinations. Asterisks denote statistical difference with respect to $W T$ at $p \leq 0.05$.

one NAC transcription factor activated by JA (denominated JA2), can induce NCED1 expression and that the JA2-NCED1 transcriptional module might be also monitoring the endogenous ABA status. Full activation of the JA2-NCED1 module by dehydration requires a basal level of $\mathrm{ABA}$. These results indicate a feedback between JA and ABA where OPR3 and NCED1 genes may have different role depending on the plant organ.

Previous studies revealed that SA treatment contributes to improve acclimation to salt stress in arabidopsis, wheat and tomato (Shakirova et al., 2003; Singh and Gautam, 2013) although contradictory results suggest a negative effect of this hormone (Borsani et al., 2001). The hormonal patterns obtained in this research showed that SA is involved in the early stages of drought response. Furthermore, expressions of ICS and PAL1 under water stress demonstrate that SA biosynthesis is activated in all groups of plants. In addition, the high expression of PAL1 in roots and leaves of $f l c$ mutants together with the decrease of SA levels in $f l c$ plants treated with exogenous $\mathrm{ABA}$ indicate that ABA may reduce SA levels by modulating PAL1 expression. Previous reports in tomato, bean and soybean have described a partial repression of PAL activity in leaves by increased ABA levels under biotic stress (Ward et al., 1989; De Meyer et al., 1999; Audenaert et al., 2002). Data obtained in this work show that ABA negatively regulates SA levels in roots and leaves of tomato also under water stress conditions.

On the other hand, the transient accumulation of JA and SA as an early response to stress could be related to an adaptive process. These results are consistent with those obtained in tomato after infestation by Bemisia tabaci (Rodríguez-Álvarez et al., 2015). Moreover, the high JA content in NahG stressed roots with respect to $\mathrm{MM}$ and the high SA content in spr2 stressed roots with respect to CSL, support the assumption that there is a coordinated action between both hormones and an antagonistic effect between JA and SA depending on levels of each hormone.

Overall, the results presented in this work indicate that water deficit induced a transient accumulation of JA and SA in roots and leaves during early events of signaling and that both hormones are involved in ABA biosynthesis. This study expands the evidence of a complex interaction between JA and ABA during water stress that modulates the levels of both hormones in roots and leaves. Finally, accumulation of SA 
suggests that this hormone can elicit plant-adaptive responses to drought. A future challenge is to uncover the key elements that connect the JA-SA crosstalk and how they work at the molecular level.

\section{AUTHOR CONTRIBUTIONS}

ML and VM performed the experiments at the greenhouse and laboratory. JC performed analysis at the laboratory. AG supervised the research. All authors contributed to write the manuscript.

\section{REFERENCES}

Audenaert, K., De Meyer, G. B., and Höfte, M. M. (2002). Abscisic acid determines basal susceptibility of tomato to Botrytis cinerea and suppresses salicylic acid- dependent signaling mechanisms. Plant Physiol. 128, 491-501. doi: 10.1104/pp.010605

Avila, C. A., Arévalo-Soliz, L. M., Jia, L., Navarre, D. A., Chen, Z., Howe, G. A., et al. (2012). Loss of function of fatty acid desaturase 7 in tomato enhances basal aphid resistance in a salicylate-dependent manner. Plant Physiol. 158, 2028-2041. doi: 10.1104/pp.111.191262

Borsani, O., Valpuesta, V., and Botella, M. A. (2001). Evidence for a role of salicylic acid in the oxidative damage generated by $\mathrm{NaCl}$ and osmotic stress in Arabidopsis seedlings. Plant Physiol. 126, 1024-1030. doi: 10.1104/pp.126.3.1024

Brossa, R., López-Carbonell, M., Jubany-Marí, T., and Alegre, L. (2011). Interplay between abscisic acid and jasmonic acid and its role in water-oxidative stress in wild-type, ABA-deficient, JA-deficient, and Ascorbate-deficient Arabidopsis plants. J. Plant Growth Regul. 30, 322-333. doi: 10.1007/s00344-0119194-Z

Burbidge, A., Grieve, T. M., Jackson, A., Thompson, A., McCarty, D. R., and Taylor, I. B. (1999). Characterization of the ABA-deficient tomato mutant notabilis and its relationship with maize Vp14. Plant J. 17, 427-431. doi: 10.1046/j.1365-313X.1999.00386.x

Chen, Z., Zheng, Z., Huang, J., Lai, Z., and Fan, B. (2009). Biosynthesis of salicylic acid in plants. Plant Signal. Behav. 4, 493-496. doi: 10.4161/psb.4.6.8392

Daszkowska-Golec, A., and Szarejko, I. (2013). Open or close the gate-stomata action under the control of phytohormones in drought stress conditions. Front. Plant Sci. 4:138. doi: 10.3389/fpls.2013.00138

De Meyer, G., Audenaert, K., and Höfte, M. (1999). Pseudomonas aeruginosa 7NSK2-induced systemic resistance in tobacco depends on in planta salicylic acid accumulation but is not associated with PR1a gene-expression. Eur. J. Plant Pathol. 105, 513-517 doi: 10.1023/A:1008741015912

De Ollas, C., Arbona, V., and Gómez-Cadenas, A. (2015). Jasmonoyl isoleucine accumulation is needed for abscisic acid build-up in roots of Arabidopsis under water stress conditions. Plant Cell Environ. 38, 2157-2170. doi: 10.1111/pce. 12536

De Ollas, C., Hernando, B., Arbona, V., and Gómez-Cadenas, A. (2013). Jasmonic acid transient accumulation is needed for abscisic acid increase in citrus roots under drought stress conditions. Physiol. Plant. 147, 296-306. doi: 10.1111/j.1399-3054.2012.01659.x

De Torres Zabala, M., Bennett, M. H., Truman, W. H., and Grant, M. R. (2009). Antagonism between salicylic and abscisic acid reflects early hostpathogen conflict and moulds plant defence responses. Plant J. 59, 375-386. doi: 10.1111/j.1365-313X.2009.03875.x

Du, M., Zhai, Q., Deng, L., Li, S., Li, H., Yan, L., et al. (2014). Closely related NAC transcription factors of tomato differentially regulate stomatal closure and reopening during pathogen attack. Plant Cell 26, 3167-3184. doi: $10.1105 /$ tpc. 114.128272

Durgbanshi, A., Arbona, V., Pozo, O., Miersch, O., Sancho, J. V., and GómezCadenas, A. (2005). Simultaneous determination of multiple phytohormones

\section{ACKNOWLEDGMENTS}

This work was supported by the Spanish Ministerio de Ciencia e Innovación through grants $\mathrm{N}^{\circ}$ AGL2013-42038R. Hormonal profiles were performed at Instrumental central facilities (SCIC) of Universitat Jaume I.

\section{SUPPLEMENTARY MATERIAL}

The Supplementary Material for this article can be found online at: http://journal.frontiersin.org/article/10.3389/fpls.2015. 00997

in plant extracts by liquid chromatography-electrospray tandem mass spectrometry. J. Agr. Food Chem. 53, 8437-8442. doi: 10.1021/jf050884b

Fang, Y., and Xiong, L. (2015). General mechanisms of drought response and their application in drought resistance improvement in plants. Cell. Mol. Life Sci. 72, 673-689. doi: 10.1007/s00018-014-1767-0

Fujita, Y., Fujita, M., Shinozaki, K., and Yamaguchi-Shinozaki, K. (2011). ABAmediated transcriptional regulation in response to osmotic stress in plants. J. Plant Res. 124, 509-525. doi: 10.1007/s10265-011-0412-3

Gómez-Cadenas, A., Arbona, V., Jacas, J., Primo-Millo, E., and Talon, M. (2002). Abscisic acid reduces leaf abscission and increases salt tolerance in citrus plants. J. Plant Growth Regul. 21, 234-240. doi: 10.1007/s00344-002-0013-4

Grebner, W., Stingl, N. E., Oenel, A., Mueller, M. J., and Berger, S. (2013). Lipoxygenase6-dependent oxylipin synthesis in roots is required for abiotic and biotic stress resistance of Arabidopsis. Plant Physiol. 161, 2159-2170. doi: 10.1104/pp.113.214544

Harb, A., Krishnan, A., Ambavaram, M. M. R., and Pereira, A. (2010). Molecular and physiological analysis of drought stress in Arabidopsis reveals early responses leading to acclimation in plant growth. Plant Physiol. 154, 1254-1271. doi: 10.1104/pp.110.161752

Hayat, Q., Hayat, S., Irfan, M., and Ahmad, A. (2010). Effect of exogenous salicylic acid under changing environment: a review. Environ. Exp. Bot. 68, 14-25. doi: 10.1016/j.envexpbot.2009.08.005

Iuchi, S., Kobayashi, M., Taji, T., Naramoto, M., Seki, M., and Kato, T. (2001). Regulation of drought tolerance by gene manipulation of 9-cis-epoxycarotenoid dioxygenase, a key enzyme in abscisic acid biosynthesis in Arabidopsis. Plant J. 27, 325-333. doi: 10.1046/j.1365-313x.2001.01096.x

Jia, C., Zhang, L., Liu, L., Wang, J., Li, C., and Wang, Q. (2013). Multiple phytohormone signalling pathways modulate susceptibility of tomato plants to Alternaria alternata f. sp. lycopersici. J. Exp. Bot. 64, 637-650. doi: $10.1093 /$ jxb/ers360

Kazan, K., and Manners, J. M. (2008). Jasmonate signaling: toward an integrated view. Plant Physiol. 146, 1459-1468. doi: 10.1104/pp.107.115717

Li, C., Liu, G. I., Xu, C., Lee, I., Bauer, P., Ling, H.-Q, et al. (2003). The tomato suppressor of prosystemin-mediated responses 2 gene encodes a fatty acid desaturase required for the biosynthesis of jasmonic acid and the production of a systemic wound signal for defense gene expression. Plant Cell. 15, 1646-1661. doi: $10.1105 /$ tpc.012237

López-Climent, M. F., Arbona, V., Peìrez-Clemente, R. M., and GoìmezCadenas, A. (2008). Relationship between salt tolerance and photosynthetic machinery performance in citrus. Environ. Exp. Bot. 62, 176-184. doi: 10.1016/j.envexpbot.2007.08.002

Mur, L. A. J., Kenton, P., Atzorn, R., Miersch, O., and Wasternack, C. (2006). The outcomes of concentration-specific interactions between salicylate and jasmonate signaling include synergy, antagonism, and oxidative stress leading to cell death. Plant Physiol. 140, 249-262. doi: 10.1104/pp.105. 072348

North, H. M., De Almeida, A., Boutin, J. P., Frey, A., To, A., Botran, L., et al. (2007). The Arabidopsis ABA-deficient mutant aba4 demonstrates that the major route for stress-induced ABA accumulation is via neoxanthin isomers. Plant J. 50, 810-824. doi: 10.1111/j.1365-313X.2007.03094.x 
Ntatsi, G., Savvas, D., Huntenburg, K., Druege, U., and Schwarz, D. (2014). A study on $\mathrm{ABA}$ involvement in the response of tomato to suboptimal root temperature using reciprocal grafts with notabilis, a null mutant in the ABA-biosynthesis gene LeNCED1. Environ. Exp. Bot. 97, 11-21. doi: 10.1016/j.envexpbot.2013.09.011

Pfaffl, M. W., Horgan, G. W., and Dempfle, L. (2002). Relative expression software tool (REST) for group-wise comparison and statistical analysis of relative expression results in real-time PCR. Nucleic Acids Res. 30:e36. doi: 10.1093/nar/30.9.e36

Rodríguez-Álvarez, C. I., López-Climent, M. F., Gómez-Cadenas, A., Kaloshian, I., Muñiz, M., and Nombela, G. (2015). Salicylic acid is required for 1 Mi-1-mediated resistance of tomato to whitefly Bemisia tabaci, but not for basal defence to this insect pest. Bull. Entomol. Res. 2, 1-9. doi: $10.1017 /$ S0007485315000449

Sagi, M., Fluhr, R., and Lips, S. (1999). Aldehyde oxidase and xanthine dehydrogenase in a flacca tomato mutant with deficient abscisic acid and wilty phenotype. Plant Physiol. 120, 571-577. doi: 10.1104/pp.120.2.571

Savchenko, T., Kolla, V. A., Wang, C. Q., Nasafi, Z., Hicks, D. R., Phadungchob, B., et al. (2014). Functional convergence of oxylipin and abscisic acid pathways controls stomatal closure in response to drought. Plant Physiol. 164, 1151-1160. doi: 10.1104/pp.113.234310

Seki, M., Umezawa, T., Urano, K., and Shinozaki, K. (2007). Regulatory metabolic networks in drought stress responses. Curr. Opin. Plant Biol. 10, 296-302. doi: 10.1016/j.pbi.2007.04.014

Seo, M., and Koshiba, T. (2002). Complex regulation of ABA biosynthesis in plants. Trends Plant Sci. 7, 41-48. doi: 10.1016/S1360-1385(01)02187-2

Seo, M., Peeters, A. J. M., Koiwai, H., Oritani, T., Marion-poll, A., Zeevaart, J. A. D., et al. (2000). The Arabidopsis aldehyde oxidase 3 (AAO3) gene product catalyzes the final step in abscisic acid biosynthesis in leaves. Proc. Natl. Acad. Sci. U.S.A. 97, 12908-12913. doi: 10.1073/pnas.220426197

Shakirova, F. M., Sakhabutdinova, A. R., Bezrukova, M. V., Fatkhutdinova, R. A., and Fatkhutdinova, D. R. (2003). Changes in the hormonal status of wheat seedlings induced by salicylic acid and salinity. Plant Sci. 164, 317-322. doi: 10.1016/S0168-9452(02)00415-6

Singh, B., and Usha, K. (2003). Salicylic acid induced physiological and biochemical changes in wheat seedlings under water stress. Plant Growth Regul. 39, 137-141. doi: 10.1023/A:1022556103536

Singh, P. K., and Gautam, S. (2013). Role of salicylic acid on physiological and biochemical mechanism of salinity stress tolerance in plants. Acta Physiol. Plant. 35, 2345-2353. doi: 10.1007/s11738-013-1279-9

Smekalova, V., Doskocilová, A., Komis, G., and Samaj, J. (2014). Crosstalk between secondary messengers, hormone and MAPK modules during abiotic stress signalling in plants. Biotechnol. Adv. 32, 2-11. doi: 10.1016/j.biotechadv.2013.07.009
Szepesi, A., Csiszár, J., Gémes, K., Horváth, E., Horváth, F., Simon, M. L., et al. (2009). Salicylic acid improves acclimation to salt stress by stimulating abscisic aldehyde oxidase activity and abscisic acid accumulation, and increases $\mathrm{Na}^{+}$ content in leaves without toxicity symptoms in Solanum lycopersicum L. J. Plant Physiol. 166, 914-925. doi: 10.1016/j.jplph.2008.11.012

Taylor, I. B., Linforth, R. S. T., Al-Naieb, R. J., Bowman, W. R., and Marples, B. A. (1988). The wilty tomato mutants flacca and sitiens are impaired in the oxidation of ABA-aldehyde to ABA. Plant Cell Environ. 11, 739-745. doi: 10.1111/j.1365-3040.1988.tb01158.x

Taylor, I. B., Sonneveld, T., Bugg, T. D. H., and Thompson, A. J. (2005). Regulation and manipulation of the biosynthesis of abscisic acid, including the supply of xanthophyll precursors. J. Plant Growth Regul. 24, 253-273. doi: 10.1007/s00344-005-0070-6

Turner, N. C. (1981). Techniques and experimental approaches for the measurement of plant water status. Plant Soil. 58, 339-366. doi: 10.1007/BF02180062

Vicente-Serrano, S. M. (2007). Evaluating the impact of drought using remote sensing in a Mediterranean, semi-arid region. Nat. Hazards 40, 173-208. doi: 10.1007/s11069-006-0009-7

Ward, E. W. B., Cahill, D. M., and Bhattacharyya, M. (1989). Abscisic acid suppression of phenylalanine ammonia lyase activity and mRNA, and resistance of soybeans to Phytophthora megaspermaf. sp. Glycinea. Plant Physiol. 91, 23-27. doi: 10.1104/pp.91.1.23

Wasternack, C., and Hause, B. (2013). Jasmonates: biosynthesis, perception, signal transduction and action in plant stress response, growth and development. Ann. Bot. 111, 1021-1058. doi: 10.1093/aob/ mct067

Yesbergenova, Z., Yang, G., Oron, E., Soffer, D., Fluhr, R., and Sagi, M. (2005). The plant Mo-hydroxylases aldehyde oxidase and xanthine dehydrogenase have distinct reactive oxygen species signatures and are induced by drought and abscisic acid. Plant J. 42, 862-876. doi: 10.1111/j.1365-313X.2005.02 422.x

Conflict of Interest Statement: The authors declare that the research was conducted in the absence of any commercial or financial relationships that could be construed as a potential conflict of interest.

Copyright $(2015$ Muñoz-Espinoza, López-Climent, Casaretto and Gómez-Cadenas. This is an open-access article distributed under the terms of the Creative Commons Attribution License (CC BY). The use, distribution or reproduction in other forums is permitted, provided the original author(s) or licensor are credited and that the original publication in this journal is cited, in accordance with accepted academic practice. No use, distribution or reproduction is permitted which does not comply with these terms. 